\title{
Contribución de las áreas verdes urbanas a la regulación del balance de agua en Santiago del Estero, Argentina
}

\author{
Urban green areas contribution to water soil partitioning \\ in Santiago del Estero City, Argentina \\ Juan Pablo Argañaraz ${ }^{a *}$, Guido Lorenz ${ }^{b}$ \\ *Autor de correspondencia: ${ }^{a}$ Universidad Nacional del Centro de la Provincia de Buenos Aires, \\ Instituto de Hidrología de Llanuras, Azul, Argentina, tel.: 54-02281-15537108, argajuan@yahoo.com.ar \\ bUniversidad Nacional de Santiago del Estero, Facultad de Ciencias Forestales, Santiago del Estero, Argentina.
}

\begin{abstract}
SUMMARY
The soil's role in water flow partitioning is considerably modified in urban environments due to pavement and buildings, which interfere with the infiltration process. In this context, green areas gain importance as water infiltration spots. The aim of this research was to evaluate the contribution of soils of Francisco de Aguirre Park to water flow partitioning in Santiago del Estero city. To fulfill this, hydraulic soil properties such as sorptivity and hydraulic conductivity were determined in situ with a tension disc infiltrometer. Results indicated that different uses of the park affect significantly the hydraulic properties: areas used in a controlled way (using footpaths) exhibited high hydraulic conductivity values, whereas areas under intensive use (sports practicing, vehicle traffic) showed low values of this property. Finally, the comparison of soil's water infiltration capacities with intensity-duration-frequency curves of precipitation indicated that the soils of the park effectively contribute to water soil partitioning in Santiago del Estero city.
\end{abstract}

Key words: urban soils, infiltration, tension disc infiltrometer, hydraulic conductivity.

\section{RESUMEN}

El rol del suelo como regulador del balance de agua se encuentra considerablemente alterado en ambientes urbanos a causa del pavimento y las edificaciones, que interfieren con el proceso de infiltración. En este contexto, las áreas verdes adquieren importancia al actuar como focos de absorción de agua. El objetivo del presente trabajo fue evaluar la contribución de los suelos del parque Francisco de Aguirre a la regulación del balance de agua de la ciudad de Santiago del Estero, Argentina. Para ello se determinaron las propiedades hidráulicas del suelo, sorptividad y conductividad hidráulica, a partir de ensayos de infiltración in situ, utilizando un infiltrómetro de disco de tensión. Los resultados indicaron que los diferentes usos del parque inciden significativamente en los valores de las propiedades hidráulicas: mientras que las zonas utilizadas de manera ordenada (respetando senderos y canteros) presentaron valores de conductividad hidráulica altos, aquellos sectores sometidos a usos intensivos (prácticas deportivas, tránsito vehicular) tuvieron valores bajos de esta propiedad. Finalmente, la comparación de las capacidades de captación de agua con las curvas de intensidad-duración-recurrencia de las lluvias indicaron que los suelos del parque contribuyen efectivamente a la regulación del balance de agua en la ciudad de Santiago del Estero.

Palabras clave: suelos urbanos, infiltración, infiltrómetro de disco de tensión, conductividad hidráulica.

\section{INTRODUCCIÓN}

El suelo es un cuerpo natural y dinámico que desempeña numerosas funciones en los ecosistemas terrestres (Schlichting 1978, Blume 1990, Doran y Parkin 1994). Entre las funciones propuestas por Schlichting (1978) y Blume (1990) se destaca la de regulación del balance de agua, que consiste en la captación y distribución de los aportes en distintos compartimentos del ecosistema, siendo el proceso de infiltración fundamental para el desempeño de tal función. En el ambiente urbano, el rol del suelo como regulador del balance de agua se encuentra considerablemente alterado (Corbin McGriff Jr 1972). La impermeabilización con pavimento y edificaciones (Pitt et al. 1999, Yusof et al. 2005), sumada a la frecuente compactación de los suelos expuestos (NRCS 2000), limitan cuantiosamente la infiltración, aumentando así la escorrentía y los riesgos de erosión e inundación (Pedron et al. 2004, Gregory et al. 2006). En este contexto, adquieren gran importancia las áreas cuyos suelos están expuestos, como es el caso de las áreas verdes y jardines domésticos, al actuar como focos de infiltración.

En la ciudad de Santiago del Estero (Argentina) la gestión del agua de precipitación no es un problema resuelto. Como consecuencia, se produce acumulación de agua en las zonas más bajas, luego de las lluvias más abundantes. 
Contribuyen a esta problemática la intensidad de las precipitaciones (Basán Nickisch 1992), un sistema de desagües pluviales inadecuado (Roldán 2000ab) y la escasez de áreas verdes que actúen como focos de infiltración.

Entre las áreas verdes de la ciudad, se destaca el parque Francisco de Aguirre por su extensión y uso popular. El mismo ocupa un área de 450 ha aproximadamente y está sometido a una gran variedad de usos, principalmente recreativos. La gran superficie del parque da lugar a suponer que éste cumple un rol destacado en la gestión del agua de precipitación de la ciudad, el cual, a su vez, puede resultar afectado por los usos a los que está sometido (Scheyer y Hipple 2005). Por ello, el objetivo de este trabajo fue analizar la contribución de los suelos del parque Francisco de Aguirre a la regulación del balance de agua en Santiago del Estero.

\section{MÉTODOS}

Área de estudio. La ciudad de Santiago del Estero (latitud: $27^{\circ} 46^{\prime} \mathrm{S}$, longitud: $\left.64^{\circ} 18^{\prime} \mathrm{O}\right)$ se sitúa en el noroeste de Argentina, sobre la margen derecha del río Dulce. El clima es templado cálido, con precipitaciones concentradas en el semestre cálido que alcanzan una media anual de $554 \mathrm{~mm}$ (Boletta et al. 1992). El parque Francisco de Aguirre (figura 1) nació en 1903 como resultado del drenaje, terraplenado y posterior plantación de eucaliptos sobre un brazo muerto del río Dulce que constituía un importante foco palúdico
(Álvarez 1941, El Liberal 1948). Años después, en 1929, la construcción de la avenida Costanera, con el objetivo de contener las crecidas del río, terminó la influencia de éste sobre los suelos ubicados hacia el oeste de la obra. Actualmente, el parque ocupa un área de 450 ha aproximadamente, que incluye espacios arbolados de carácter público (parque propiamente dicho) y una gran superficie concedida a clubes y entidades de bien público. Los espacios de carácter público son utilizados para diferentes fines, principalmente recreativos, con gran afluencia de personas. La vegetación presente es muy diversa, con ejemplares de la flora nativa y exótica de formas biológicas y fenología variadas. Se destacan eucaliptos (Eucalyptus spp.) y casuarinas (Casuarina spp.) por su amplia distribución, acompañadas de lapachos (Tabebuia spp.), algarrobos (Prosopis spp.), palmeras (familia Palmae) y coníferas varias, entre otras especies.

Los suelos del parque Francisco de Aguirre se caracterizan por una notable intervención antrópica. Se trata de suelos jóvenes (edad $\approx 100$ años), resultantes del depósito de materiales (terraplenado) a principios del 1900, sobre el suelo aluvial existente. El espesor del relleno es de $70 \mathrm{~cm}$ aproximadamente, con una textura franco arenosa. Debajo del mismo se encuentra el horizonte $2 \mathrm{Ah}$, que es el horizonte superior de la antigua superficie de origen fluvial, cuya textura es franco limosa. El número 2 indica una discontinuidad litológica, es decir, el origen diferente de los materiales. Según IUSS Grupo de Trabajo WRB (2007), le corresponde la denominación Hypo-Technic Regosol (Eutric, Transportic) over Haplic Fluvisol (Eutric).

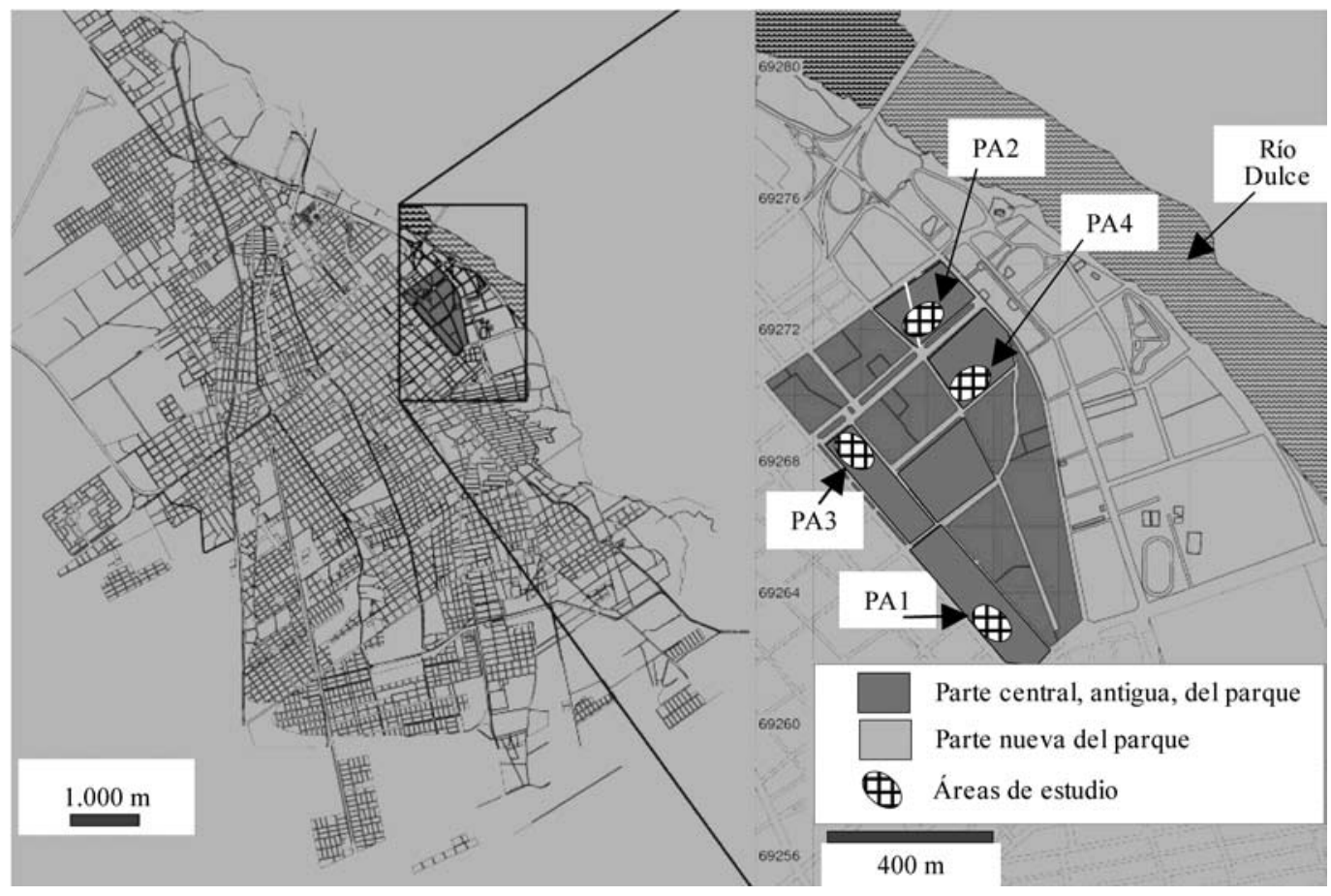

Figura 1. Área de estudio (sistema de referencia WGS84, sistema de coordenadas Gauss-Krüger) (Municipalidad de Santiago del Estero 2006).

Study area (WGS84 Reference system, Gauss-Krüger coordinate system) (Municipalidad de Santiago del Estero 2006). 
Unidades de estudio. El estudio se llevó a cabo en las áreas arboladas públicas del parque Francisco de Aguirre. Éstas se clasificaron en tres categorías (niveles 1 al 3) en función del grado de ordenamiento, manejo e intensidad de uso. Se seleccionaron cuatro unidades (PA 1 al 4), abarcando así la diversidad de ambientes del parque, cubriendo aproximadamente 2/3 de la superficie arbolada pública (cuadro 1). Además, se estudiaron dos subunidades con uso intensivo: senderos y cancha de fútbol, a fin de analizar situaciones extremas. Si bien actualmente la superficie ocupada por éstas es $<10 \%$ del área de estudio, muchas veces son establecidas por los usuarios del parque sin ningún tipo de control o planificación por parte de las autoridades. La cobertura arbórea es de 20 a $40 \%$ en PA1, de 40 a $60 \%$ en PA3 y PA4 y de 60 a $80 \%$ en PA2. Se considera que las unidades PA1, PA3 y PA4 tienen un alto grado de ordenamiento, al tener delimitados senderos y canteros que promueven el uso cuidadoso por quienes concurren al parque. Por el contrario, la falta de este tipo de límites en PA2 promueve usos considerados intensivos, que incluyen tránsito peatonal, vehicular y práctica de deportes, al igual que en PASe y PA6. La pendiente general es nula y sólo se manifiestan desniveles al pie de la avenida Costanera (entre las partes nueva y antigua del Parque Aguirre, figura 1), en senderos que están delimitados por canteros de mayor altura y sitios de uso intensivo como son canchas de fútbol, juegos infantiles y pistas de salud. Existe un sistema de evacuación de agua por medio de bocas de tormenta en calles aledañas, que en ocasiones se encuentran total o parcialmente obstruidas por residuos domésticos y hojarasca.

Metodología. Las propiedades hidráulicas del suelo son necesarias para predecir el flujo de agua en el ambiente
(White y Perroux 1987). Parte de este flujo está comprendido en el proceso de infiltración, el cual puede ser descrito a partir de dos propiedades hidráulicas: la conductividad hidráulica y la sorptividad (Zhang 1997ab, Wang et al. 1998). Estas propiedades, a su vez, se encuentran influenciadas por el sistema poroso del suelo, que depende de la textura y la estructura.

Por ello, se determinó la conductividad hidráulica $(K)$ y la sorptividad $(S)$, a fin de describir el proceso de infiltración, y se analizaron las propiedades del sistema poroso y la materia orgánica del suelo, para explicar y facilitar la interpretación de los resultados.

Determinación de las propiedades hidráulicas. Las mediciones de infiltración se realizaron in situ con un infiltrómetro de disco de tensión Guelph (Soil Moisture Equipment, Santa Barbara, California) (figura 2). La aplicación de diferentes potenciales negativos (tensiones) al agua del instrumento permite excluir del proceso de infiltración una cierta fracción de poros (Elrick y Reynolds 1992, White et al. 1992). Esto resulta deseable por el gran aporte de los macroporos al flujo de agua (Watson y Luxmoore 1986) y la sensibilidad de esta propiedad al manejo o factores ambientales (Perroux y White 1988). La tensión aplicada fue de $\psi=-0,5 \mathrm{~cm}$ de columna de agua, excluyendo así los macroporos de diámetro $>6 \mathrm{~mm}$, entre los que se incluyen túneles de hormigueros o grietas que pueden ser muy localizados y no representan las condiciones generales de la matriz del suelo. La duración de las mediciones fue máximamente de $30 \mathrm{~min}$, llegando hasta $40 \mathrm{~min}$ en PA6 y PASe. El pleno contacto entre el infiltrómetro y el suelo, fundamental durante las mediciones (Perroux y White 1988), se logró utilizando una capa de arena tamizada entre 0,2 y $0,1 \mathrm{~mm}$.

Cuadro 1. Clasificación y características de las unidades de estudio.

Classification and features of the sampling units.

Categoría de

clasificación

Parque de recreación

Nivel 1

Parque de recreación Nivel 2

Parque de recreación Nivel 3

de motocross, ciclismo, tránsito de maquinarias pesadas.
Comprende los senderos que atraviesan las unidades PA3 y PA4. Uso intensivo: tránsito peatonal y vehicular (motocicletas, bicicletas).
Unidades

de estudio

Cobertura arbórea y herbácea abundante. Manejo intensivo que incluye riego mediante aspersores de impulso y recolección de hojarasca. Alto grado de ordenamiento: senderos y canteros delimitados que promueven uso responsable $\mathrm{u}$ ordenado.

Cobertura arbórea y herbácea abundante. Manejo moderado que incluye riego y recolección de hojarasca en algunos sectores. Alto grado de ordenamiento: senderos y canteros delimitados que promueven uso responsable u ordenado.

Cobertura arbórea abundante, manto herbáceo discontinuo. Manejo de baja intensidad: sin riego, sólo recolección de hojarasca que posteriormente se quema en superficies pequeñas. Bajo grado de ordenamiento: senderos sin delimitar. Uso intensivo: carreras

PA2

PA3

PA1, PA4

Senderos Uso intensivo de alto impacto.

PASe

Cancha de fútbol

PA6 


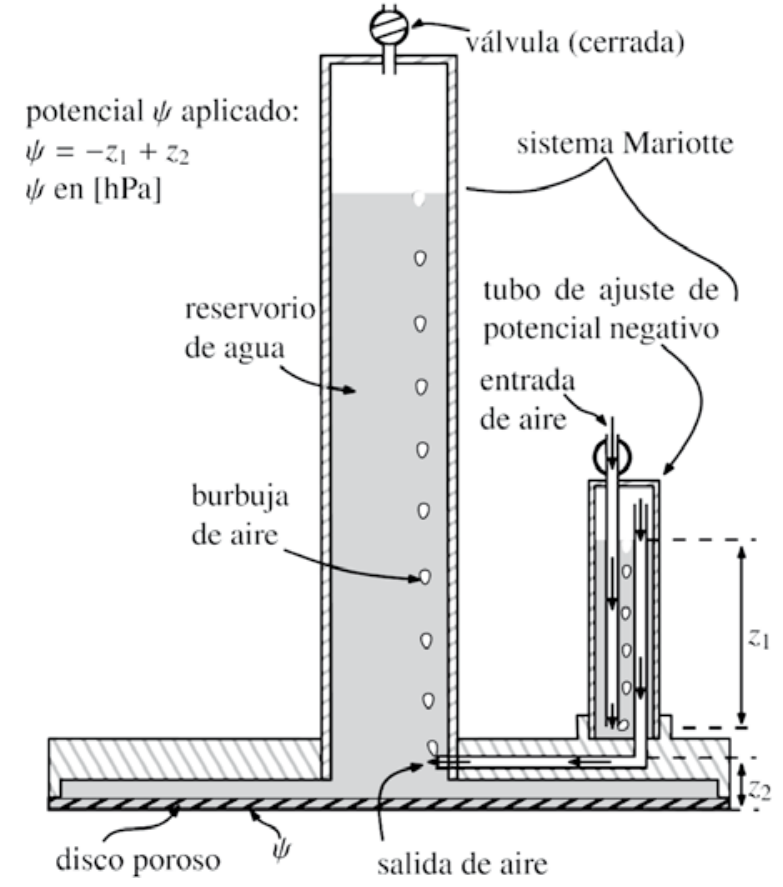

Figura 2. Infiltrómetro de tensión (modificado de White et al. 1992).

Tension infiltrometer (Modified from White et al. 1992).

Las propiedades hidráulicas se determinaron a partir de la técnica analítica propuesta por Vandervaere et al. (1997, 2000ab). Ésta emplea la ecuación [1], basada en la de Philip (1957), para describir el proceso de infiltración tridimensional desde un infiltrómetro de disco en el estado no estacionario:

$$
I=C_{1} \sqrt{t}+C_{2} t
$$

Donde $I=$ Infiltración acumulada, $t=$ tiempo, $C_{1}=$ coeficiente relacionado a la sorptividad $(S), C_{2}=$ coeficiente relacionado a la conductividad hidráulica $(K)$.

$$
\begin{gathered}
C_{1}=S \\
C_{2}=\frac{2-\beta}{3} K+\frac{\gamma S^{2}}{R\left(\theta_{0}-\theta_{n}\right)}
\end{gathered}
$$

Donde $\beta, \gamma=$ constantes iguales a 0,6 y 0,75 , respectivamente (Vandervaere et al. 2000ab); $R=$ radio del disco, $\theta_{n}=$ contenido volumétrico de agua inicial del suelo, $\theta_{0}=$ contenido volumétrico de agua final del suelo.

Utilizando las ecuaciones [2] y [3] para reemplazar $C_{1}$ y $C_{2}$ en [1], el modelo de infiltración se puede expresar de la siguiente manera [4]:

$$
I=S \sqrt{t}+\left[\frac{2-\beta}{3} K+\frac{\gamma S^{2}}{R\left(\theta_{0}-\theta_{n}\right)}\right] \cdot \mathrm{t}
$$

En este modelo, $S \sqrt{t}$ es el término capilaridad vertical, $[(2-\beta) / 3] \cdot K t$ es el término gravitacional y $\left\{\gamma S^{2} /\left[R\left(\theta_{0}-\theta_{n}\right)\right]\right\} \cdot t$ es el término capilaridad lateral. El dominio de uno u otro término en el proceso de infiltración puede ser identificado al comparar el valor de la sorptividad del suelo con el parámetro $S_{\text {opt }}\left(\mathrm{L} \mathrm{T}^{-1 / 2}\right)$ (Vandervaere et al. 2000b) [5]:

$$
S_{o p t}=\sqrt{\frac{R\left(\theta_{0}-\theta_{n}\right)(2-\beta) K}{3 \gamma}}
$$

Donde $S_{\text {opt }}$ es el valor de sorptividad en el que, para un conjunto de valores de $R, \theta_{0}-\theta_{n}, \beta, K$ y $\gamma$, los términos gravitacional y capilaridad lateral tienen igual peso en el proceso de infiltración. Las situaciones pueden ser tres: i) si $S<S_{\text {opt }}$, el flujo es dominado por la gravedad y las condiciones son favorables para una estimación precisa de $K$, pero no así de $S$; ii) cuando $S>S_{\text {opt }}$, el flujo es dominado por la capilaridad lateral y no es posible estimar $K$ con precisión, ni $S$, que está relacionado a la capilaridad vertical, y iii) si $S \approx S_{\text {opt }}$, los términos gravitacional y capilaridad lateral tienen igual peso en el proceso de infiltración y es posible una estimación confiable de $K$ y $S$.

Para la determinación de los coeficientes $C_{1}$ y $C_{2}$, Vandervaere et al. (2000ab) proponen una rectificación de la ecuación [1], que consiste en la diferenciación de la infiltración acumulada con respecto a la raíz cuadrada del tiempo. Esta técnica se denomina "método de rectificación diferenciada" (método DL, differentiated linearization) y la ecuación resultante es [6]:

$$
\frac{d I}{d \sqrt{t}}=C_{1}+2 C_{2} \sqrt{t}
$$

El cociente $d I / d \sqrt{t}$ es aproximado por [7]:

$$
\frac{d I}{d \sqrt{t}} \approx \frac{\Delta I}{\Delta \sqrt{t}}=\frac{I_{i+1}-I_{i}}{\sqrt{t_{i+1}}-\sqrt{t_{i}}}(i=1 \ldots n-1)
$$

Donde $n=$ número de datos, $i=$ número de intervalo de tiempo.

El valor correspondiente de $\sqrt{t}$ es calculado como la media geométrica [8]:

$$
\sqrt{t}=\left[\sqrt{t_{i} t_{i+1}}\right]^{1 / 2} \quad(i=1 \ldots n-1)
$$

De acuerdo con la ecuación [6], el gráfico $\frac{\Delta I}{\Delta \sqrt{t}}$ versus $\sqrt{t}$ debe ser lineal, con $C_{1}$ igual a la ordenada en el origen y $C_{2}$ igual a la mitad de la pendiente. Luego, el cálculo de los coeficientes $C_{1}$ y $C_{2}$ se realiza por regresión lineal sobre la porción ascendente. En la práctica, dicho gráfico presenta una porción inicialmente descendente, que 
corresponde a la infiltración en el material de contacto y una subsiguiente, linealmente ascendente, que corresponde a la infiltración en el suelo. Esta característica del gráfico permite identificar y eliminar la influencia que la capa de contacto tiene en los primeros estadios del proceso de infiltración, como consecuencia del agua que se almacena en la misma. Asimismo, existen otras causas que pueden desviar el gráfico de la linealidad, entre ellas: la interrupción del contacto hidráulico entre el suelo y el disco por oscilaciones del infiltrómetro o la presencia de rocas o capas impermeables (Vandervaere et al. 2000a). Esta sensibilidad de la técnica representa una ventaja respecto de los métodos basados en la infiltración acumulada ya que no sólo permite eliminar la influencia de la capa de contacto, sino también identificar la porción del conjunto de datos que no se adecuan al modelo (no son lineales), facilitando su exclusión (Minasny y McBratney 2000, Vandervaere et al. 2000a). En función de ello, la estimación de los parámetros $C_{1}$ y $C_{2}$ en cada ensayo se realizó sobre el conjunto de datos consecutivos que presentaba un comportamiento lineal ascendente.

Una vez determinados $C_{1}$ y $C_{2}$, existen distintos métodos para estimar $S$ y $K$ en función de la relación $S / S_{\text {opt }}$. Mediciones previas en el área de estudio indicaron la pertenencia al dominio gravitacional de la mayoría de los puntos medidos $\left(S<S_{\text {opt }}\right)$. En estas condiciones, Vandervaere et al. (2000b) sugieren la determinación de $S$ y $K$ con el denominado Método Simple, que emplea un único tamaño de disco y un único valor de tensión en cada punto de medición. Luego, $S$ y $K$ se determinan despejando las ecuaciones [2] y [3].

Aplicación práctica de los valores de $K$. De acuerdo con Wilson y Luxmoore (1988) se puede asumir que la conductividad hidráulica es igual al valor de la tasa de infiltración cuando el flujo de agua en el suelo ha alcanzado el estado estacionario. La consideración de los valores de $K$ como valores mínimos de capacidad de infiltración tiene un carácter conservador en un estudio de riesgos de inundación, dado por el margen de "tolerancia" que se obtiene al despreciar el aporte de la sorptividad. Tomando esta asunción, se cuantificaron las capacidades de infiltración ponderada en PA2, PA3 y PA4 a partir de los valores de conductividad hidráulica determinados y se compararon con las curvas de intensidad-duración-recurrencia de las precipitaciones de la ciudad (Basán Nickisch 1992), a fin de evaluar el aporte que estas unidades hacen a la regulación del balance de agua en el sistema urbano. Para ello se generaron mapas detallados de cada unidad, estratificando cada una según los tipos de uso y a cada uno se le asignó una tasa de infiltración. Se aplicaron factores de corrección en los sectores con pendiente, considerando un rango entre 0 (pendiente vertical) y 1 (sin pendiente). Partiendo de 1 , el valor se reduce en 0,1 por cada $10^{\circ}$ de pendiente. A modo de ejemplo, los bordes del terraplén que limita
PA2 tienen una pendiente de $60^{\circ}$, por lo que se le aplicó un factor de 0,4. A las superficies selladas se les asignó una capacidad nula.

Descripción del sistema poroso. A la par de cada medición de infiltración, se tomaron dos muestras de suelo de $100 \mathrm{~cm}^{3}$, una de ellas sin disturbar. Con ésta se determinó la densidad aparente (Dap) por gravimetría (Blake y Hartge 1986) y la distribución de los tamaños de poros a través de la curva de retención de agua (Klute 1986) aplicando potenciales pF: 0,$6 ; 1,8 ; 2,5$ y 4,2 (4, 63, 330 $15.000 \mathrm{hPa}$ o centímetros de columna de agua) ${ }^{1}$, asumiendo una relación $\theta(\psi)$ uniforme hasta $1 \mathrm{~m}$ de profundidad. La función de retención de agua fue calculada utilizando el modelo de Van Genuchten (1980). A partir de la curva se estimó la capacidad de campo $(C C$; límite de $C C: \mathrm{pF}=$ $1,8)$ y el agua útil $(A U)$ del suelo. Con la segunda muestra se determinó el contenido de agua del suelo al inicio del ensayo de infiltración $\left(\theta_{n}\right)$. El contenido volumétrico de agua luego del ensayo $\left(\theta_{0}\right)$ se estimó a partir de la curva de retención de agua, para una columna de agua de $0,5 \mathrm{~cm}$ (valor de tensión aplicado).

También se determinó la materia orgánica del suelo (MOS) por oxidación en húmedo con dicromato de potasio, calentamiento a $120^{\circ} \mathrm{C}$ y detección espectrofotométrica de $\mathrm{Cr}^{3+}$ (Schlichting et al. 1995).

Diseño de muestreo y análisis estadísticos. Se realizó un muestreo semisistemático, disponiendo en cada unidad dos transectas paralelas de $60 \mathrm{~m}$ de longitud separadas entre sí a $40 \mathrm{~m}$, colocando el origen al azar. Sobre éstas se ubicaron cuatro puntos de muestreo cada $20 \mathrm{~m}$ (tamaño de muestra $n=8$ ). En PA6, la separación entre transectas y puntos fue de $4 \mathrm{~m}$ y en PASe se realizaron mediciones sobre los senderos que atravesaban las transectas en PA3 y PA4.

Los análisis estadísticos utilizados incluyen: prueba de normalidad de Shapiro-Wilk, prueba de homogeneidad de variancias de Levene, análisis de la variancia ANOVA y prueba de Tukey para la comparación por pares. En caso de no cumplimiento de los supuestos del ANOVA, se recurrió a una transformación de la variable (Sachs 1978, Montgomery 1993). Los análisis estadísticos se realizaron con el software R 2.5.1 ( $\mathrm{R}$ Development Core Team 2007).

\section{RESULTADOS}

Aspectos metodológicos. La gráfica $\frac{\Delta I}{\Delta \sqrt{t}}$ versus $\sqrt{t}$ descrita por Vandervaere et al. (2000a) con una porción inicialmente descendente, correspondiente a la infiltración

$1 \quad 1 \mathrm{~cm}$ de columna de agua $=0,981 \mathrm{hPa}$. 
en el material de contacto y la subsiguiente linealmente ascendente, correspondiente a la infiltración en el suelo, sólo se verificó en 15 de los 48 puntos analizados en este estudio (figura 3). De ellos, la mayoría se presentó en los sitios de menor conductividad hidráulica, donde el contraste de esta propiedad entre el material de contacto y el suelo es mayor. Además, en 20 casos se observó una fase de transición entre la infiltración correspondiente a la capa de arena y la correspondiente al suelo (figura 3), de los cuales más de la mitad pertenecían a PA6 y PASe, sugiriendo que las fuerzas capilares del material de contacto y del suelo eran semejantes en estos sitios (Bagarello e Iovino 2003). El resto de las mediciones presentó un comportamiento lineal ascendente desde el comienzo del ensayo. La exclusión de los datos que no presentaban comportamiento lineal ascendente resultó en estimaciones de $C_{1}$ y $C_{2}$ sobre lapsos de tiempo entre 12 y 30 minutos, con tres casos de 5,8 y 9 minutos.

Caracterización del sistema poroso. De acuerdo con Adhoc-AG Boden (2005), el volumen total de poros es escaso en PA6, regular en PA2 y PASe y alto para los sitios PA1, PA3 y PA4 (cuadro 2). En cuanto al volumen de macroporos totales, $M T,(\varnothing \geq 10 \mu \mathrm{m})$, los sitios con mayor valor y próximos al $30 \%$, son PA1, PA3 y PA4. Luego siguen PA2 y PASe con volúmenes en torno al $20 \%$ y en

Cuadro 2. Valores medios ( \pm desviación estándar) de porosidad total $(P)$, macroporos totales $(M T,>10 \mu \mathrm{m})$, macroporos anchos $(M A=C A,>50 \mu \mathrm{m})$, densidad aparente $($ Dap $)$ y materia orgánica $(M O S)$ por sitio $(\mathrm{n}=8)$.

Mean values $( \pm$ standard deviation) of total porosity $(\mathrm{P})$, total macropores $(M T,>10 \mu \mathrm{m})$, coarse macropores $(M A=C A,>50 \mu \mathrm{m})$, bulk density (Dap) and soil organic matter $(M O S)$ per site $(\mathrm{n}=8)$.

\begin{tabular}{lccccc}
\hline \multicolumn{1}{c}{ Sitio } & $\begin{array}{c}P \\
{[\% \text { volumen }]}\end{array}$ & $\begin{array}{c}M T \\
{[\% \text { volumen }]}\end{array}$ & $\begin{array}{c}M A(C A) \\
{[\% \text { volumen }]}\end{array}$ & $\begin{array}{c}\text { Dap } \\
{\left[\mathrm{g} \mathrm{cm}^{-3}\right]}\end{array}$ & $\begin{array}{c}M O S \\
{[\%]}\end{array}$ \\
\hline PA1 & $52,9 \pm 4,9$ & $30,3 \pm 5,5 \mathrm{a}$ & $13,8 \pm 4,6$ & $1,25 \pm 0,14$ & $2,0 \pm 0,4$ \\
PA2 & $45,8 \pm 9,1$ & $21,2 \pm 5,6 \mathrm{~b}$ & $12,2 \pm 4,6$ & $1,43 \pm 0,25$ & $2,9 \pm 1,3$ \\
PA3 & $59,7 \pm 4,8$ & $28,5 \pm 4,3 \mathrm{a}$ & $17,8 \pm 4,5$ & $1,07 \pm 0,13$ & $2,7 \pm 0,5$ \\
PA4 & $52,1 \pm 3,8$ & $32,3 \pm 2,8 \mathrm{a}$ & $13,1 \pm 3,3$ & $1,27 \pm 0,11$ & $1,7 \pm 0,8$ \\
PA6 & $38,2 \pm 3,1$ & $12,6 \pm 3,3 \mathrm{c} \dagger$ & $4,1 \pm 1,6$ & $1,64 \pm 0,11$ & - \\
PASe & $42,4 \pm 3,5$ & $19,6 \pm 4,6 \mathrm{~b}$ & $8,6 \pm 3,4$ & $1,57 \pm 0,10$ & - \\
\hline
\end{tabular}

n: tamaño de muestra. $\dagger \mathrm{n}=7$ por descarte de una muestra. Letras distintas indican diferencias significativas (Tukey, $P<0,05$ ).
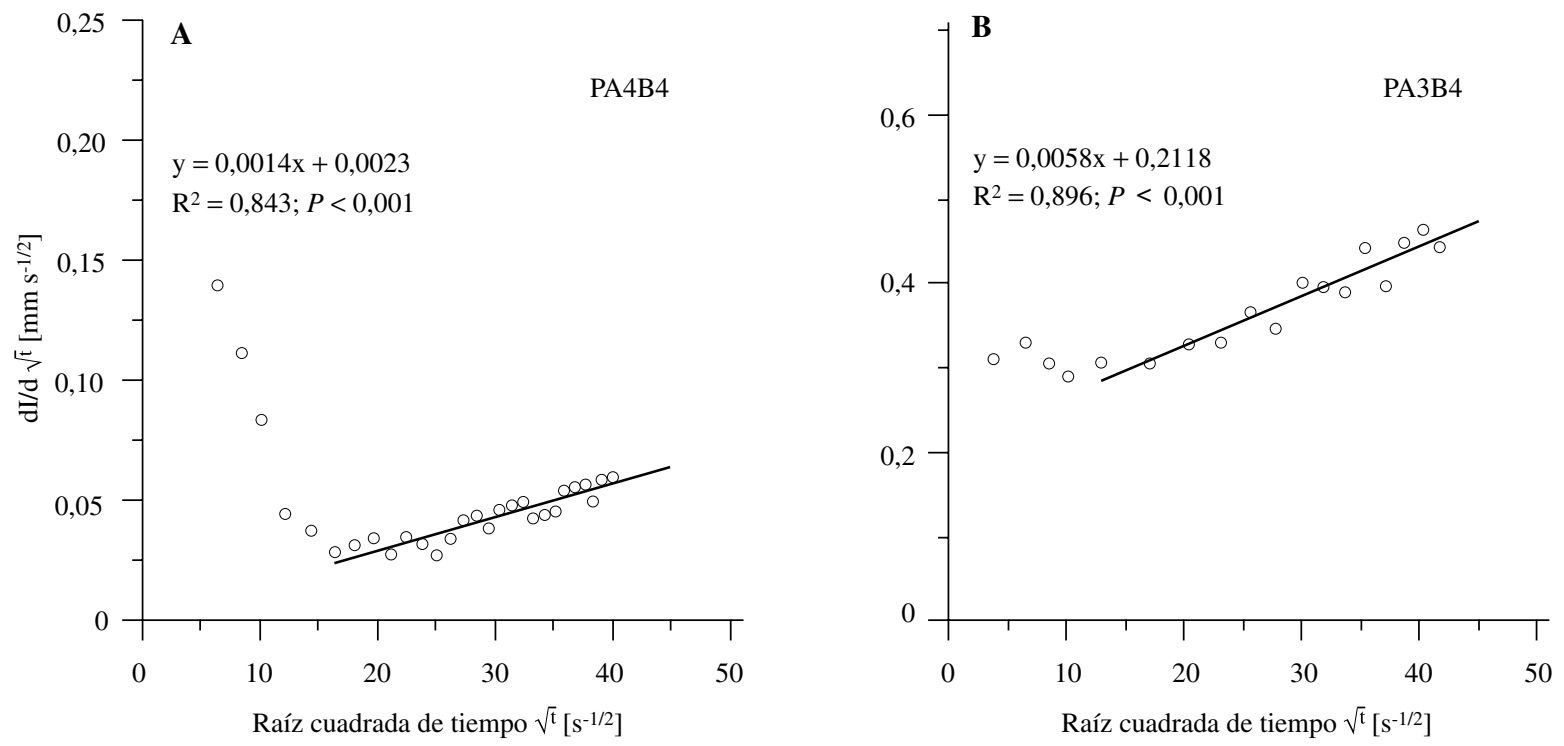

Figura 3. A) Curva típica con sección descendente al inicio, debido a la influencia de la capa de contacto y B) curva con fase de transición.

A) Typical curve with an early decreasing portion due to the influence of the contact layer and B) curve with a transition phase. 
último lugar PA6 con 12,6\%. El ANOVA indicó diferencias significativas en el porcentaje de $M T$ entre los sitios $(P<0,001)$ (cuadro 2$)$, coincidiendo con el agrupamiento mencionado de acuerdo a sus valores medios.

De acuerdo con Schlichting et al. (1995), la proporción de macroporos anchos $(\varnothing \geq 50 \mu \mathrm{m}$, también llamada capacidad de aire del suelo, $C A$ ) en PA1, PA2, PA3 y PA4 es alta, mientras que en PASe es regular y en PA6 escasa. La densidad aparente, según AG Bodenkunde (1982), es muy baja en PA3, baja en PA1 y PA4 y media en los sitios de mayor Dap: PA2, PA6 y PASe.

En cuanto al contenido de materia orgánica, los suelos de PA1, PA2 y PA3 se consideran humosos (2-4\%) y los de PA4 como escasamente humosos (1-2\%) (Schlichting et al. 1995). Si bien la $M O S$ mejora las características del sistema poroso al promover la formación y estabilidad de los agregados (Gavande 1991), la asociación con las propiedades del sistema poroso sólo se verificó con $M A$ y el volumen de macroporos finos $(10 \mu \mathrm{m}<\varnothing<50 \mu \mathrm{m})$, aunque los coeficientes de correlación fueron bajos: $\mathrm{r}=0,42(P<0,05)$ y $\mathrm{r}=0,36(P<0,05)$, respectivamente.

Resumiendo, los sitios que tienen un uso ordenado (PA1, PA3 y PA4) mantienen mejores condiciones del sistema poroso que aquellas unidades que tienen usos más intensivos y no controlados (PA2, PA6 y PASe).

En cuanto a las capacidades de almacenamiento de agua del suelo, las unidades PA1 y PA3 tienen capacidades de campo altas (390-520 $\mathrm{L} \mathrm{m}^{-3}$ ), mientras que en los sitios restantes son consideradas regulares (260-390 L m-3) según AG Boden (1996). El agua útil se considera extremadamente alta en PA1, PA3 y PA4 $\left(>270 \mathrm{~L} \mathrm{~m}^{-3}\right)$ y en las demás unidades muy alta (200-270 $\left.\mathrm{L} \mathrm{m}^{-3}\right)$, según Schlichting et al. (1995). De acuerdo con estas evaluaciones, se puede afirmar que los suelos estudiados presentan condiciones favorables para la regulación del balance de agua.

Propiedades hidráulicas. El dominio de las fuerzas gravitacionales en 41 de los 48 puntos medidos $\left(S<S_{\text {opt }}\right)$ permitió estimaciones de conductividad hidráulica precisas, aunque no así de sorptividad. Debido a esta carencia de precisión, las estimaciones de $S$ no son expuestas en este trabajo.

Los valores de $K$ se analizaron a partir de la media geométrica, dado que los valores originales no cumplían los requisitos para ser analizados con la estadística paramétrica. Los valores medios de $K$ van desde $3,0 \mathrm{~mm} \mathrm{~h}^{-1}$ (PASe) hasta 36,9 $\mathrm{mm} \mathrm{h}^{-1}$ (PA4) (cuadro 3, figura 4). De acuerdo con Schlichting et al. (1995) y AG Boden (1996), los sitios PA1, PA3 y PA4 tienen valores de $K$ altos, mientras que en PA2 éste es regular y en PA6 y PASe se consideran bajos.

La comparación de la conductividad hidráulica por sitios se debió realizar con la variable transformada $\log (10 K)$, respondiendo a la distribución teórica logarítmica mencionada por Russo y Bresler (1981) y Wu et al. (1999). El ANOVA indicó diferencias significativas entre sitios $(P<0,001)$. La comparación por pares señaló semejanzas
Cuadro 3. Media geométrica y función de transformación de la conductividad hidráulica $K$ por sitio $(\mathrm{n}=8)$.

Geometric mean and transformation function for hydraulic conductivity $K$ per site $(\mathrm{n}=8)$.

\begin{tabular}{ccc}
\hline Sitio & Media geométrica & $\log (10 K)$ \\
\hline PA1 & 28,8 & $2,46 \mathrm{a}$ \\
PA2 & 10,2 & $2,01 \mathrm{~b}$ \\
PA3 & 17,3 & $2,24 \mathrm{ab}$ \\
PA4 & 36,9 & $2,57 \mathrm{a}$ \\
PA6 & 3,2 & $1,51 \mathrm{c}$ \\
PASe & 3,0 & $1,48 \mathrm{c}$ \\
\hline
\end{tabular}

n: tamaño de muestra. $K\left[\mathrm{~mm} \mathrm{~h}^{-1}\right]$. Letras distintas indican diferencias significativas (Tukey, $P<0,05$ ).

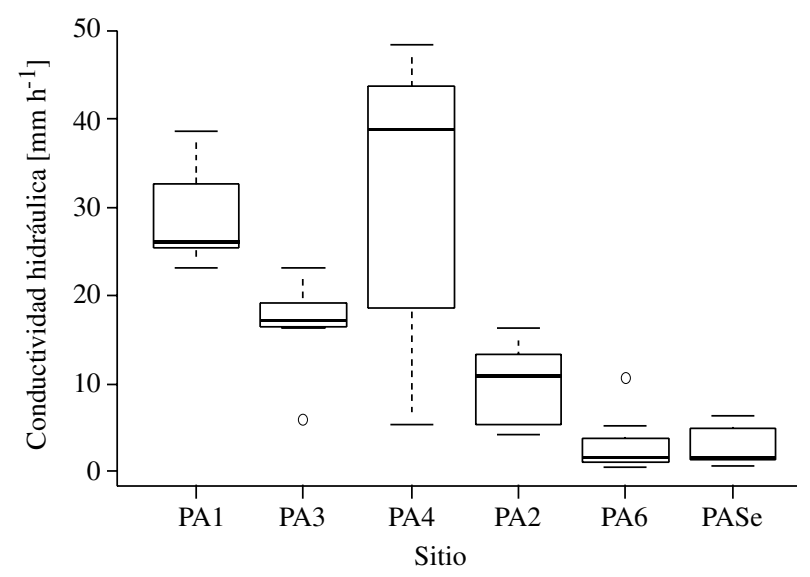

Figura 4. Gráficos de cajas de los valores de conductividad hidráulica $K$ por sitio.

Box plot of the hydraulic conductivity values $K$ per site.

entre los sitios PA1, PA3 y PA4 (cuadro 3), coincidiendo con sus valores de conductividad hidráulica altos. La similitud entre PA6 y PASe indica que los usos intensivos a los que están sometidos afectan de igual manera a esta variable, disminuyendo sus valores.

Con respecto a la relación entre las propiedades del sistema poroso y la conductividad hidráulica, se encontró una mayor asociación entre $K$ y los macroporos totales, explicando $M T$ cerca del $50 \%$ de la variabilidad del modelo lineal $\left(\mathrm{R}^{2} \approx 0,47, P<0,001\right)$ y exponencial $\left(\mathrm{R}^{2} \approx 0,50\right.$, $P<0,001)$ (figura 5).

Aplicación práctica de los valores de K. La unidad PA4 presentó la mayor capacidad de infiltración por unidad de área (cuadro 4). Esto se explica por su valor de $K$ alto y la baja importancia relativa de la superficie ocupada por senderos $(\approx 5 \%)$. El valor marcadamente inferior de PA3 se explica tanto por el valor de $K$ menor al de PA4 como por la presencia de dos edificaciones y una gran superficie 

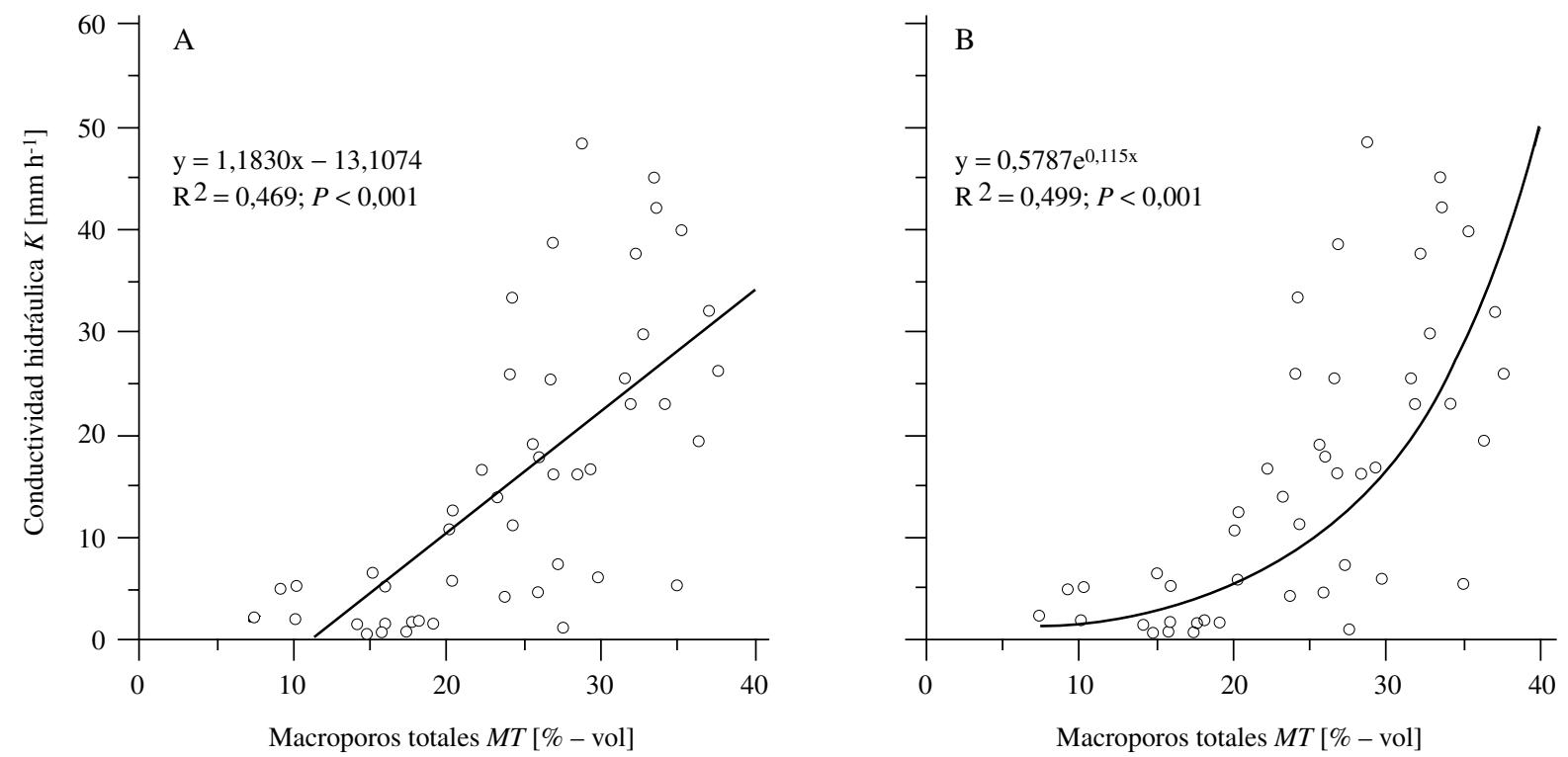

Figura 5. Regresión lineal (A) y exponencial (B) entre conductividad hidráulica y macroporos totales. Linear (A) and exponential (B) regressions between hydraulic conductivity and total macropores.

ocupada por senderos (15 a $20 \%$ ), algunos de los cuales se encuentran pavimentados. El valor de $K$ considerado regular y la existencia de una cancha de fútbol en PA2 resultan en el valor más bajo de capacidad de infiltración por unidad de área.

Cuadro 4. Capacidad de infiltración ponderada por unidad de área en tres sitios para un período de 60 minutos.

Weighted infiltration capacity per unit area for a $60 \mathrm{~min}$ period of three sites.

\begin{tabular}{cccc}
\hline Sitio & Área total & $\begin{array}{c}\text { Capacidad de } \\
\text { infiltración } \\
{\left[\mathrm{dm}^{3}\right]}\end{array}$ & $\begin{array}{c}\text { Capacidad de } \\
\text { infiltración/área } \\
{\left[\mathrm{dm}^{3} \mathrm{~m}^{-2}\right]}\end{array}$ \\
\hline PA2 & 39.112 & 344.045 & 8,8 \\
PA3 & 36.403 & 464.471 & 12,8 \\
PA4 & 43.181 & 1.334 .203 & 30,9 \\
\hline
\end{tabular}

Las intersecciones entre las curvas correspondientes a las tasas de infiltración de las unidades PA2, PA3 y PA4 con las funciones que corresponden a precipitaciones de distintas duraciones (figura 6) (Basán Nickisch 1992), marcan la recurrencia máxima para la cual cada unidad tiene capacidad de absorber el agua sin producir anegamiento. De ello se desprende que en PA4 habrá acumulación de agua en superficie para las precipitaciones máximas de 5 y $30 \mathrm{~min}$, en tanto que para las de 120 min esto ocurrirá para episodios máximos que ocurren cada seis años. Las unidades PA2 y PA3 tendrán acumulación de agua libre en todas las circunstancias analizadas, a excepción de episodios anuales de 120 min de duración (figura 6).

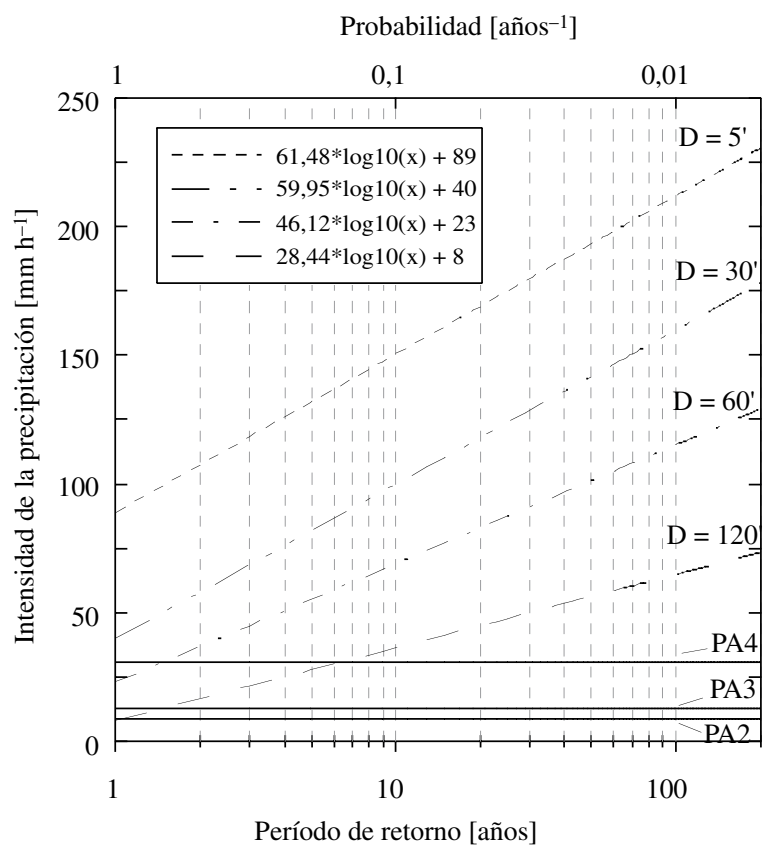

Figura 6. Curvas de intensidad-duración y recurrencia de las precipitaciones de Santiago del Estero (1956-1986) y La Banda (1962-1981) por el método de Gumbel (1958), basadas en datos de Basán Nickisch (1992). D: duración. Las líneas horizontales representan la capacidad de infiltración ponderada $\left[\mathrm{mm} \mathrm{h}^{-1}\right]$ para tres sitios.

Intensity-duration and recurrence curves for precipitations of Santiago del Estero (1956-1986) and La Banda (1962-1981) by Gumbel's method (1958) based on Basán Nickisch (1992) data. D: duration. Horizontal lines represent weighted infiltration capacity $\left[\mathrm{mm} \mathrm{h}^{-1}\right]$ of the three sites. 
Considerando períodos de recurrencia de hasta 10 años, los tiempos de inundación del suelo van desde $<1,5 \mathrm{~h}$ en las condiciones más favorables (PA4), aumentando hasta $7 \mathrm{~h}$ en condiciones más desfavorables (PA2) (cuadro 5). Sin embargo, en las subunidades que presentan menor capacidad de infiltración (PA6 y PASe), estos tiempos aumentan, pudiendo alcanzar las 6,5 h en eventos anuales y hasta $22 \mathrm{~h}$ para precipitaciones de recurrencia decádica (cuadro 5).

Cuadro 5. Tiempo [min] que el suelo permanece inundado para precipitaciones (pp.) de diferente duración [min] y recurrencia en tres unidades y dos subunidades del parque Francisco de Aguirre.

Period of time [min] that soils remain flooded by precipitations (pp.) of variable duration [min] and recurrence of three sites and two sub-units of Aguirre Park.

\begin{tabular}{crrrrrrrrr}
\hline Duración pp. & \multicolumn{1}{c}{5} & \multicolumn{2}{c}{$30^{\prime}$} & \multicolumn{2}{c}{$60^{\prime}$} & \multicolumn{2}{c}{$120{ }^{\prime}$} \\
\hline $\begin{array}{c}\text { Recurrencia } \\
\text { [años] }\end{array}$ & 1 & 10 & 1 & 10 & 1 & 10 & 1 & 10 \\
\hline PA2 & 46 & 83 & 106 & 313 & 97 & 417 & 0 & 371 \\
PA3 & 30 & 55 & 64 & 206 & 48 & 268 & 0 & 218 \\
PA4 & 9 & 20 & 9 & 68 & 0 & 76 & 0 & 20 \\
\hline PA6 & 136 & 240 & 351 & 929 & 378 & 1.273 & 185 & 1.251 \\
PASe & 140 & 247 & 362 & 957 & 391 & 1.313 & 194 & 1.292 \\
\hline
\end{tabular}

\section{DISCUSIÓN}

Aspectos metodológicos. Bagarello e Iovino (2003) reportaron la existencia de una fase de transición entre la infiltración correspondiente a la capa de contacto y la correspondiente al suelo en ensayos de campo. Estos autores descartaron las mediciones que presentaban tal comportamiento, argumentando que la dificultad de separar la infiltración en uno y otro medio limita la aplicación del método de rectificación diferenciada. En este trabajo no se hizo tal descarte, sino que se calcularon los coeficientes $C_{1}$ y $C_{2}$ excluyendo los puntos iniciales de la infiltración, cuando se observó una discontinuidad en el alineamiento, de manera que la curva permitiera el ajuste lineal del modelo. En todos estos casos, el tiempo restante del experimento fue lo suficientemente largo para que se pudieran estimar los parámetros.

Los tiempos empleados en este trabajo para la estimación de los parámetros $C_{1}$ y $C_{2}$ superan los 3 a 5 min sugeridos por Vandervaere et al. (2000b), sin embargo, se consideró más apropiado contar con un mayor volumen de datos en las estimaciones. Por el contrario, la recomendación de Bagarello e Iovino (2003) de realizar mediciones de 60 min no se consideró conveniente, ante la posibilidad de alcanzar el estado de flujo estacionario, como reportaron Ela et al. (1992) y Bodhinayake et al. (2004) en suelos de textura más fina que los del parque
Francisco de Aguirre. Si se alcanzara tal estado, el uso del modelo de infiltración de Vandervaere et al. (2000a) no sería válido, dado que este sólo es aplicable en la fase no estacionaria del flujo.

La rigurosidad del método DL para el análisis de los datos dio lugar al descarte de 18 mediciones que proporcionaban estimaciones negativas de $C_{1}$ o $C_{2}$, lo cual no tendría una explicación física posible. La subunidad con más casos fue el de los senderos, debiendo descartarse el $50 \%$ de las mediciones. La causa de estas anomalías podría deberse a la falta de cumplimiento de los supuestos del modelo de infiltración (homogeneidad e isotropismo del sistema poroso), ya que algunas de las muestras recolectadas presentaban diferencias de material a escasa profundidad. La utilización de técnicas de análisis basadas en la infiltración acumulada no hubiera permitido identificar tales anomalías en el proceso. De esta forma, el empleo del método DL permite asegurar que el ensayo se desarrolló de acuerdo con los supuestos del modelo de infiltración, dando validez a la utilización de éste para determinar las propiedades hidráulicas.

Propiedades hidráulicas. Estudios de infiltración en ambientes urbanos reportaron estimaciones semejantes de $K$ en suelos arenosos, con valores desde $<1 \mathrm{~mm} \mathrm{~h}^{-1}$, en suelos altamente disturbados, hasta $38 \mathrm{~mm} \mathrm{~h}^{-1}$ en suelos no disturbados (bosque) (OCSCD 2001). De manera similar, Pitt et al. (2002) determinaron tasas de infiltración desde 3,6 $\mathrm{mm} \mathrm{h}^{-1}$, en suelos arenosos compactados, hasta $33,0 \mathrm{~mm} \mathrm{~h}^{-1}$ en suelos no compactados.

En cuanto a la relación de $K$ con las propiedades del sistema poroso, la mayor asociación entre $K$ y $M T$ se debe a que la permeabilidad aumenta exponencialmente con un aumento en el tamaño de poros (Nelson y Baver 1940), en concordancia con la ley de Poiseuille. Es decir, que si bien la porosidad representa la totalidad del espacio que puede ser ocupado por agua, el diámetro y la distribución del tamaño de poros determinarán la velocidad del flujo (Potter et al. 1988). De acuerdo con el potencial aplicado, participaron en el flujo poros de hasta $6 \mathrm{~mm}$ de diámetro, por lo que la contribución de los macroporos en el proceso debió ser importante. Además, Lutz y Leamer (1939) afirman que la permeabilidad aumenta rápidamente con un aumento en el porcentaje de macroporos, lo que explica la mejor adecuación del modelo exponencial, aunque este ajuste sea sólo ligeramente mejor.

La explicación solamente parcial de la variabilidad de $K$ a partir de los $M T$ puede deberse a que el flujo de agua no sólo depende del diámetro del poro, sino también de la continuidad (Bouma 1982, Ela et al. 1992), longitud (Smettem 1986), forma y tortuosidad del poro, entre otros aspectos (Arya et al. 1999). Además, la existencia de costras biológicas o minerales en la superficie, observadas en algunos puntos, pudo disminuir el tamaño de poros participantes en el flujo (Bouma 1982) y, en algunos casos, 
la presencia de material orgánico pudo tener un efecto de repelencia al agua (Wahl et al. 2003), disminuyendo las tasas de infiltración en el suelo. Esto último reafirma la importancia de preservar las condiciones superficiales del suelo mediante la utilización de una capa de contacto al momento de medir infiltración, a fin de representar en forma fehaciente lo que ocurre en la realidad.

Relación entre las propiedades del suelo y las características y usos de las unidades de estudio. Los sitios con mayores valores de $K, \mathrm{PA} 1, \mathrm{PA} 3$ y PA4 tienen valores de $P, M T$, $M A$ y Dap "favorables". Estas condiciones no sorprenden considerando el uso ordenado y de bajo impacto que se hace de los mismos, favorecido por la delimitación de senderos y canteros. El menor valor de $K$ de PA3, respecto de PA1 y PA4, podría deberse al riego, que en ocasiones favorece la formación de costras biológicas o minerales.

Las características del sistema poroso observadas en PA2, PA6 y PASe muestran cómo el uso intensivo compacta y degrada el suelo. Estas unidades reflejan que la compactación disminuye el volumen de poros grandes y que esta disminución es acompañada por una reducción de la conductividad hidráulica del suelo, en concordancia con Zhang et al. (2006).

Capacidad de regulación del balance de agua de los suelos del parque Francisco de Aguirre. Los tiempos de inundación estimados a nivel de unidad indican que el parque Francisco de Aguirre contribuye efectivamente a la regulación del balance de agua en Santiago del Estero. Sin embargo, al analizar las subunidades senderos y cancha de fútbol, la situación es menos favorable. Éstos no sólo tienen capacidades de infiltración bajas, sino que generalmente constituyen áreas deprimidas que reciben un aporte adicional de agua proveniente de los alrededores. Esto provoca que los tiempos de anegamiento sean mayores a los estimados, como se pudo apreciar in situ luego de lluvias intensas en que el agua persistió en superficie aún 72 horas después de las precipitaciones.

La acumulación de agua en superficie tiene consecuencias indeseables para el manejo del parque al afectar la funcionalidad del mismo como sitio de esparcimiento y aumentando los riesgos de compactación (Nimmo y Akstin 1988). Estos problemas podrían agravarse de continuar la tendencia creciente de las precipitaciones anuales y la mayor frecuencia de eventos extremos registrados en los últimos años a nivel regional (Minetti y Acuña 1994).

Recomendaciones de manejo. Como se demostró anteriormente, el manejo y uso del suelo tienen gran incidencia en las propiedades del sistema poroso y en la conductividad hidráulica. Por tal motivo, se recomienda adoptar medidas que eviten la proliferación de canchas de fútbol y senderos sin planificación. Asimismo, en los últimos años se ha observado una tendencia a pavimentar senderos y calles internas del parque, práctica que produce un sellado del suelo y disminuye la superficie por donde puede infiltrar el agua de lluvia.

Si bien en este trabajo la asociación entre el contenido de materia orgánica del suelo y las propiedades del sistema poroso fue pobre o nula, sus efectos benéficos son bien conocidos (Walker y Chong 1986, Gavande 1991, Beyer et al. 1995, Pitt et al. 2002). Por tal motivo, se recomienda evitar la recolección y quema de hojarasca, a fin de aumentar el contenido de materia orgánica de los suelos.

\section{CONCLUSIONES}

Los valores de conductividad hidráulica del parque Francisco de Aguirre están relacionados con el manejo y uso de los suelos. Donde el uso es ordenado, los valores de $K$ son altos; mientras que donde el uso es intensivo, los valores son regulares a bajos.

La explicación solamente parcial de la conductividad hidráulica a partir de las propiedades del sistema poroso indica que la magnitud de $K$ depende también de otras variables y condiciones del suelo. Esto descarta la posibilidad de estimar la conductividad hidráulica a partir de las propiedades del sistema poroso en los suelos estudiados y reivindica la importancia de la medición in situ de las propiedades hidráulicas.

Los suelos del parque Francisco de Aguirre contribuyen efectivamente a la regulación del balance de agua en Santiago del Estero, al presentar capacidades de infiltración suficientes para absorber las precipitaciones esperadas en tiempos aceptables. Los tiempos de anegamiento más prolongados se presentan en aquellos sectores que están sometidos a usos intensivos y que tienen menor capacidad de infiltración de agua.

Se sugiere la adopción de medidas tendientes a mantener y mejorar la calidad del suelo en el parque Francisco de Aguirre: evitar aquellas prácticas que sellan el suelo y regular los usos de impacto negativo.

\section{AGRADECIMIENTOS}

A la Dra. Marta Pece por el asesoramiento estadístico, al Ingeniero Basán Nickisch por la bibliografía facilitada y al Ministerio de Educación, Ciencia y Tecnología de la Nación, que financió el proyecto de "Voluntariado Universitario": "Características y funciones de suelos urbanos -una contribución a la gestión urbana" (Res. N 7 SPU del 08/09/2006), en el cual se enmarcó este trabajo.

\section{REFERENCIAS}

Ad-hoc-AG Boden. 2005. Bodenkundliche Kartieranleitung. Hannover, Germany. Bundesanstalt für Geowissenschaften und Rohstoffe. 438 p. 
AG Boden. 1996. Bodenkundliche Kartieranleitung. 4ª ed. Hannover, Germany. Bundesanstalt für Geowissenschaften und Rohstoffe und Geologische Landesämter Deutschland. $392 \mathrm{p}$.

AG Bodenkunde. 1982. Bodenkundliche Kartieranleitung. $3^{\text {a }}$ ed. Hannover, Germany. Bundesanstalt für Geowissenschaften und Rohstoffe und Geologische Landesämter Deutschland. $331 \mathrm{p}$.

Álvarez A. 1941. Santiago del Estero: Ciudad de invierno. Buenos Aires, Argentina. Pesce y Cía. Impresores. 233 p.

Arya LM, FJ Leij, PJ Shouse, MTh. Van Genuchten. 1999. Relationship between the hydraulic conductivity function and the particle size distribution. Soil Sci. Soc. Am. J. 63: 1063-1070.

Bagarello V, M Iovino. 2003. Field testing parameter sensitivity of the two-term infiltration equation using differentiated linearization. Vadose Zone Journal 2: 358-367.

Basán Nickisch M. 1992. Curvas intensidad-duración-recurrencia para la ciudad de Santiago del Estero. Santiago del Estero, Argentina. Convenio Bilateral CFI-Provincia de Santiago del Estero, Área: Hidrología.

Beyer L, HP Blume, DC Elsner, A Willnow. 1995. Soil organic matter composition and microbial activity in urban soils. The Science of the Total Environment 168:267-278.

Blake GR, KH Hartge. 1986. Bulk Density. In Klute A. eds. Methods of soil analysis. Part 1: Physical and mineralogical methods. Madison, USA. Agronomy 9 ASA. ASA Inc. Publisher. p. 363-375.

Blume HP. 1990. Einführung. In Blume HP eds. Handbuch des Bodenschutzes. Landsberg, Germany. Ecomed-Verlag. p. 1-3.

Bodhinayake W, BC Si, K Norobio. 2004. Determination of hydraulic properties in sloping landscapes from tension and double-ring infiltrometers. Vadose Zone J. 3: 964-970.

Boletta PE, LR Acuña, ML Juárez de Moya. 1992. Análisis de las características climáticas de la provincia de Santiago del Estero y su comportamiento durante la sequía de la campaña agrícola 1988-1989. Santiago del Estero, Argentina. INTA-UNSE. 23 p.

Bouma J. 1982. Measuring the hydraulic conductivity of soil horizons with continuous macropores. Soil Sci. Soc. Am. J. 46: 438-441.

Corbin McGriff Jr E. 1972. The effects of urbanization on water quality. J. Environ. Qual. 1: 86-89.

Doran JW, TB Parkin. 1994. Defining and assessing soil quality. In Doran JW, DC Coleman, DF Bezdiek, BA Stewart eds. Defining soil quality for a sustainable environment. Wisconsin, USA. SSSA Special Publication 35. p. 3-21

Duchaufour P. 1987. Manual de edafología. Barcelona, España. Masson S.A. 214 p.

Ela SD, SC Gupta, WJ Rawls. 1992. Macropore and surface seal interactions affecting water infiltration into soil. Soil Sci. Soc. Am. J. 56: 714-721.

El Liberal. 1948. Número del cincuentenario. Santiago del Estero, Argentina. El Liberal. 502 p.

Elrick DE, WD Reynolds. 1992. Infiltration from constant-head permeameters and infiltrometers. In Topp GC, WD Reynolds, RE Green eds. Advances in measurement of soil physical properties: bringing theory into practice. Wisconsin, USA. SSSA Special Publication 30. p. 1-22.

Gavande SA. 1991. Física de suelos. Principios y aplicaciones. México D. F., México. Limusa. 351 p.
Gregory JH, MD Dukes, PH Jones, GL Miller. 2006. Effect of urban soil compaction on infiltration rate. Journal of Soil and Water Conservation 61(3): 117-123.

Gumbel EJ. 1958. Statistics of extremes. New York. USA. Columbia University Press. 375 p.

IUSS Working Group WRB. 2007. World reference base for soil resources 2006. First Update 2007. World Soil Resources Report No. 103. Rome, Italy. FAO. 128 p.

Klute A. 1986. Water retention: Laboratory methods. In Klute A. ed. Methods of soil analysis. Part 1: Physical and mineralogical methods. Madison, USA. Agronomy 9 ASA. ASA Inc. Publisher. p. 635-662.

Lutz JF, RW Leamer. 1939. Pore-size distribution as related to the permeability of soils. Soil Sci. Soc. Am. Proc. 4:28-31.

Minasny B, AB McBratney. 2000. Estimation of sorptivity from disc-permeameter. Geoderma 95: 305-324.

Minetti JL, LR Acuña. 1994. Régimen de variabilidad interanual de las precipitaciones en el centro-este de la provincia de Santiago del Estero. El salto climático de las décadas de 1950-60. Santiago del Estero, Argentina. Instituto Nacional de Tecnología.

Montgomery DC. 1993. Diseño y análisis de experimentos. México DF, México. Grupo Editorial Iberoamérica. 589 p.

Municipalidad de Santiago del Estero. 2006. Plano digital de la ciudad de Santiago del Estero, Argentina. Dirección de Suelos Urbanos. Responsables Técnicos: Fares V, LA Luna.

Nelson WR, LD Baver. 1940. Movement of water through soils in relation to the nature of the pores. Soil Sci. Soc. Am. Proc. 5: 69-76.

Nimmo JR, KC Akstin. 1988. Hydraulic conductivity of a sandy soil at low water content after compaction by various methods. Soil Sci. Soc. Am. J. 52:303-310.

NRCS (Natural Resources Conservation Service, US). 2000. Urban soil compaction. Soil quality-Urban technical note $\mathrm{N}^{\circ}$ 2. Consultado 4 ago. 2006. Disponible en http://soils. usda.gov/sqi/files/u02.pdf.

OCSCD (Ocean County Soil Conservation District, US). 2001. Impact of soil disturbance during construction on bulk density and infiltration in Ocean County New Jersey. 20 p. Consultado 30 Nov. 2006. Disponible en http://www.ocscd. org/soil.pdf

Pedron F de A, RS Diniz Dalmolin, AC de Azevedo, J Kaminski. 2004. Solos urbanos. Ciência Rural 34: 1647-1653.

Perroux KM, I White. 1988. Designs for disc permeameters. Soil Sci. Soc. Am. J. 52: 1205-1215.

Philip JR. 1957. The theory of infiltration: 4. Sorptivity and algebraic infiltration equations. Soil Sci. 84: 257-264.

Pitt R, J Lantrip, R Harrison. 1999. Infiltration through disturbed urban soil and compost-amended soil effects on runoff quality and quantity. U.S. Environmental Protection Agency. USA. Consultado 18 ago. 2006. Disponible en http://rpitt. eng.ua.edu/Publications/Publications.shtml.

Pitt R, S Chen, S Clark. 2002. Compacted urban soils effects on infiltration and bioretention stormwater control designs. 9th International Conference on Urban Drainage. Portland Oregon. IAHR IWA EWRI and ASCE. Portland, USA.

Potter KN, FS Carter, EC Doll.1988. Physical properties of constructed and undisturbed soils. Soil Sci. Soc. Am. J. 52: 1435-1438.

R Development Core Team. 2007. R: A language and environment for statistical computing. Vienna, Austria. R Foundation for Statistical Computing. 
Roldán CF. 2000a. Estudio hidrológico e hidráulico de la cuenca del colector Santa Fe. In Uso y preservación de los recursos hídricos en los umbrales del Siglo XXI. Memorias del XVIII Congreso Nacional del Agua. Santiago del Estero, Argentina. Universidad Nacional de Santiago del Estero. p. $65-66$.

Roldán CF. 2000b. Aplicación de paquetes de software para hidrología en el diseño del colector pluvial Colón. In Uso y preservación de los recursos hídricos en los umbrales del Siglo XXI. Memorias del XVIII Congreso Nacional del Agua. Santiago del Estero, Argentina. Universidad Nacional de Santiago del Estero. p. 67-68.

Russo D, E Bresler. 1981. Soil hydraulic properties as stochastic processes: I. An analysis of field spatial variability. Soil Sci. Soc. Am. J. 45: 682-687.

Sachs L. 1978. Estadística aplicada. Barcelona, España. Editorial Labor. $567 \mathrm{p}$.

Scheyer JM, KW Hipple. 2005. Urban soil primer. United States Department of Agriculture, Natural Resources Conservation Service, National Soil Survey Center, Lincoln, Nebraska. Consultado 11 nov. 2006. Disponible en http://soils.usda. gov/use/urban/primer.html g

Schlichting E. 1978. Funktionen von Böden in der Ökosphäre. Daten u. Dokumente Z. Umweltschutz. 22: 9-12.

Schlichting E, HP Blume, K Stahr. 1995. Bodenkundliches Praktikum. Eine Einführung in pedologisches Arbeiten für Ökologen insbesondere Land- und Forstwirte und für Geowissenschaftler. Blackwell Wissenschafts-Verlag. Berlin, Deutschland. 295 p.

Smettem KRJ. 1986. Analysis of water flow from cylindrical macropores. Soil Sci. Soc. Am. J. 50: 1139-1142.

Vandervaere JP, C Peugeot, M Vauclin, R Angulo Jaramillo, T Lebel. 1997. Estimating hydraulic conductivity of crusted soils using disc infiltrometers and minitensiometers. Journal of Hydrology 188-189: 203-223.

Vandervaere JP, M Vauclin, DE Elrick. 2000a. Transient flow from tension infiltrometers: I. The two-parameter equation. Soil Sci. Soc. Am. J. 64: 1263-1272.

Vandervaere JP, M Vauclin, DE Elrick. 2000b. Transient flow from tension infiltrometers: II. Four methods to determine sorptivity and conductivity. Soil Sci. Soc. Am. J. 64: 12721284.

Van Genuchten MTh. 1980. A closed-form equation for predicting the hydraulic conductivity of unsaturated soils. Soil Sci. Soc. Am. J. 44: 892-898.
Wahl NA, O Bens, B Schäfer, RF Hüttl. 2003. Impacts of changes in land-use management on soil hydraulic properties: hydraulic conductivity water repellency and water retention. Physics and Chemistry of the Earth 28: 1377-1387.

Walker J, SK Chong. 1986. Characterization of compacted soil using sorptivity measurements. Soil Sci. Soc. Am. J. 50: 288-291.

Wang D, SR Yates, FF Ernst. 1998. Determination of soil hydraulic properties using tension infiltrometers, time domain reflectometry and tensiometers. Soil Sci. Soc. Am. J. 62:318-325.

Watson KW, RJ Luxmoore. 1986. Estimating macroporosity in a forest watershed by use of a tension infiltrometer. Soil Sci. Soc. Am. J. 50: 578-582.

White I, KM Perroux. 1987. The use of sorptivity to determine field soil hydraulic properties. Soil Sci. Soc. Am. J. 51: 1093-1101.

White I, MJ Sully, KM Perroux. 1992. Measurements of surface-soil hydraulic properties: disk permeameters tension infiltrometers and other techniques. In Topp GC, WD Reynolds, RE Green eds. Advances in measurement of soil physical properties: bringing theory into practice. Wisconsin. USA. SSSA Special Publication 30. p. 69-104.

Wilson GV, RJ Luxmoore. 1988. Infiltration macroporosity and mesoporosity distributions on two forested watersheds. Soil Sci. Soc. Am. J. 52: 329-335.

Wu L, L Pan, J Mitchel, B Sanden. 1999. Measuring saturated hydraulic conductivity using a generalized solution for singlering infiltrometers. Soil Sci. Soc. Am. J. 63: 788-792.

Yusof MF, NA Zakaria, AA Ghani, R Abdullah, CK Chang. 2005. Infiltration study for urban soil: case studies-Butterworth and Engineering Campus Universiti Sains Malaysia. In Proceedings of the Congress-International Association for Hydraulic Research September 11-16 2005. Seoul, Korea.

Zhang R. 1997a. Determination of soil sorptivity and hydraulic conductivity from the disk infiltrometer. Soil Sci. Soc. Am. J. 61:1024-1030.

Zhang R. 1997b. Infiltration models from the disk infiltrometer. Soil Sci. Soc. Am. J. 61:1597-1603.

Zhang S, H Grip, L Lövdahl. 2006. Effect of soil compaction on hydraulic properties of two loess soils in China. Soil and Tillage Research 90: 117-125.

Recibido: 16.02.10 Aceptado: 09.08.10 